Please do not remove this page

RMIT

UNIVERSITY

\title{
A single-bit digital non-coherent baseband BFSK demodulator
}

Thompson, Adam; Hussain, Zahir; O'Shea, Peter

https://researchrepository.rmit.edu.au/esploro/outputs/9921857781501341/filesAndLinks?institution=61RMIT_INST\&index=null

Thompson, A., Hussain, Z., \& O'Shea, P. (2004). A single-bit digital non-coherent baseband BFSK

demodulator. IEEE TENCON 2004, 515-518. https://doi.org/10.1109/TENCON.2004.1414470

Published Version: https://doi.org/10.1109/TENCON.2004.1414470

Repository homepage: https://researchrepository.rmit.edu.au

(c) 2004 IEEE. Personal use of this material is permitted. However, permission to reprint/republish this material for advertising or promotional purposes or for creating new collective works for resale or redistribution to servers or lists, or to reuse any copyrighted component of this work in other works must be obtained from the IEEE.

Downloaded On 2023/04/26 23:50:23 +1000 


\title{
A SINGLE-BIT DIGITAL NON-COHERENT BASEBAND BFSK DEMODULATOR
}

\author{
Adam Charles Thompson ${ }^{\dagger}$, Zahir M. Hussain ${ }^{\dagger}$, and Peter O'Shea ${ }^{\ddagger}$ \\ ${ }^{\dagger}$ School of Electrical and Computer Engineering, \\ RMIT University, Melbourne, Victoria, Australia \\ Emails: s2114313@student.rmit.edu.au and zmhussain@ieee.org \\ ${ }^{\ddagger}$ School of Electrical and Electronic Systems Engineering, \\ Queensland University of Technology, Brisbane, Australia \\ Email: pj.oshea@qut.edu.au
}

\begin{abstract}
Recently we have presented efficient structures for filters that produce an output with a single-bit resolution. As an application and extension of these works a baseband digital single-bit non-coherent Binary Frequency Shift Keying (BFSK) demodulator is proposed. This new structure contains no multi-bit multiplications unlike traditional methods of digital BFSK demodulation. The single-bit BFSK demodulator is then simulated using an additive white gaussian noise channel. As a benchmark the single-bit BFSK demodulator and the theoretical non-coherent BFSK demodulator bit errors are calculated and compared.
\end{abstract}

\section{INTRODUCTION}

The proliferation of Sigma-Delta data converters currently available on the market suggests that it is a popular choice of conversion element. The oversampled single-bit format produced by sigma-delta analogto-digital conversion is generally resampled to the Nyquist rate then filtered to produce the traditional multi-bit output [1]. This filtering removes the quantization noise introduced by the coarse, often single-bit, quantization. Once filtered, traditional digital-signalprocessing operations are used to manipulate the signal.

However, the advantages of keeping a data converters output in the single-bit format are only recently being explored [2][3][4][5]. As the single-bit format alphabet is composed of only two symbols $\{-1,1\}$, the multiplication operation becomes trivial; implementation can be realized with either a multiplexor or a couple of logic gates [6]. This is the converse of the multibit multiplication that requires complex logic. The single-bit format requires much less internal signalrouting within field programmable gate arrays (FPGA) or integrated circuits as compared to the multi-bit alternative. Finally, sigma-delta analog-to-digital converters can be made simpler as the decimation and filtering stages are no longer required. This decrease in com-

0-7803-8560-8/04/\$20.00(c)2004IEEE plexity implies reduced silicon space requirements in implementation of single-bit systems.

In this paper we propose the use of single-bit techniques to demodulate a baseband Binary Frequency Shift Keying (BFSK) signal. We assume that the signals at the input to such a demodulator are at baseband frequencies; or equivalently may have been transmitted in this form within a digital system.

Matched filters and coherent detection systems in BFSK will provide greater signal to noise ratios and hence, lower probabilities of error at the receiver. However, coherent detection normally requires phase synchronization between the carrier signal and the local oscillators at the receiver. Typically, phase synchronization circuits are required; such synchronization circuits are complex. Hence, many BFSK receivers employ a non-coherent demodulator. The proposed demodulator will be of the non-coherent type. A block diagram of a non-coherent BFSK demodulator is shown in Figure 1.

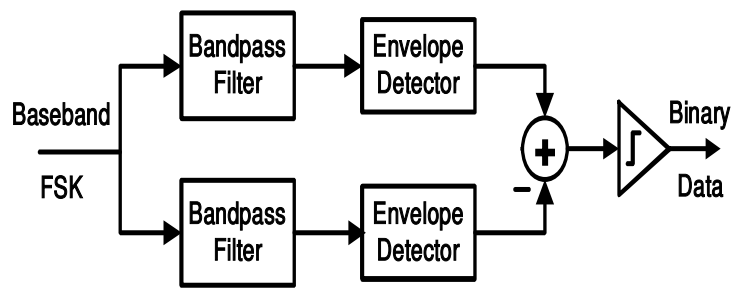

Fig. 1. Block diagram of a non-coherent BFSK demodulator.

This BFSK demodulator employs two narrowband finite impulse response (FIR) filters. The center frequencies of the filters are set to $\omega_{1}$ and $\omega_{2}$ where the basis functions generated at the transmitter are $\cos \left(\omega_{1} t\right)$ and $\cos \left(\omega_{2} t\right)$ and $t$ represents time and $\omega$ radian frequency. An envelope detector is used to generate a DC level from each narrowband filter. The resultant DC levels are then added and passed to a threshold detector that determines which level, and hence which frequency, was received.

This structure can be made more efficient through the use of single-bit filtering [2] [7] and through the 
provision of a single-bit output [3]. We propose a BFSK demodulator that can use either a single- or multi-bit word input that efficiently produces a singlebit demodulated output.

\section{THE SINGLE-BIT BFSK DEMODULATOR}

TThe structure of the proposed single-bit BFSK demodulator is shown in Figure 2. The structure is similar to the traditional non-coherent demodulator shown in Figure 1.

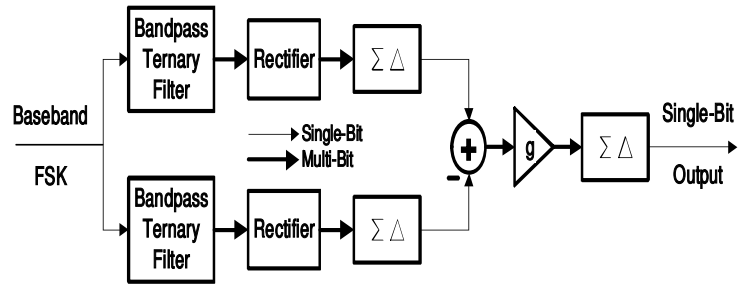

Fig. 2. Block diagram of the efficient non-coherent BFSK demodulator.

The bandpass ternary FIR filters are efficient filters that have coarsely quantized filter coefficients. The filtered signals from each frequency branch are rectified (any sample less than zero is set to zero) whilst they are in the multi-bit format produced by the ternary filter. Remodulation then puts the rectified signals into the single-bit format. This reduces the complexity of the subtraction of the two frequency branches. The subtractor output is then multiplied by a gain and remodulated to the single-bit format. The multiplication and subtraction operation can be implemented with a small lookup table to keep the system efficient.

\section{THE TERNARY FIR FILTER}

The ternary filter is an FIR filter with a reduced coefficient alphabet. The ternary filter coefficients, or taps, are restricted to the set $\{-1,0,1\}$. Mathematically, the FIR filter output $y(k)$ can be described by the convolution of the ternary taps $h_{i}$ and the input signal $x(k)$. Given $M$ as the order of the FIR filter, the output of the ternary filter would be

$$
y(k)=\sum_{i=0}^{M} h_{i} x_{k-i}, \quad h_{i} \in\{1,0,-1\} .
$$

The structure of the ternary FIR filter is shown in Figure 3. The limited tap values allow for a simple hardware implementation. Multiplication in the singlebit domain can be achieved with a couple of logic gates or a simple lookup table [6]. As for the zero in the ternary alphabet, it is simply implemented with a delay and no input to the final summer within the ternary filter is required. After many simulation studies the authors found that up to half of the tap values for simple lowpass filters could be zero. This significantly reduces the number of bits required at the output of the summer and makes ternary filters simple and efficient in hardware implementation.

The authors use Sigma-Delta modulation of a target impulse response to generate the ternary taps [2][8][9]. The double loop $\Sigma \Delta \mathrm{m}$ shown in Figure 4 was used due to its implementation simplicity. Higher order modulators will produce filters with higher stopband attenuations however, such high stopband attenuation is not needed in this application. The z-domain transfer function of the double loop $\Sigma \Delta \mathrm{m}$ is given by [1]:

$$
H(z)=G(z) z^{-1}+E(z)\left(1-2 z^{-1}+z^{-2}\right)
$$

where $G(z)$ represents the target impulse response and $E(z)$ represents the quantization noise transfer functions. The noise shaping effect of the $\Sigma \Delta \mathrm{m}$ is evident from the presence of the filtering term, $\left(1-2 z^{-1}+\right.$ $z^{-2}$ ), acting on the noise term, $E(z)$. The frequency response of the above $\Sigma \Delta \mathrm{M}$ is given by:

$$
H_{\Sigma \Delta T}\left(e^{j \Omega}\right)=G\left(e^{j \Omega}\right) e^{-j \Omega}+E\left(e^{j \Omega}\right)\left(1-2 e^{-j \Omega}+e^{-2 j \Omega}\right)
$$

where $\Omega$ is the normalized radian frequency.

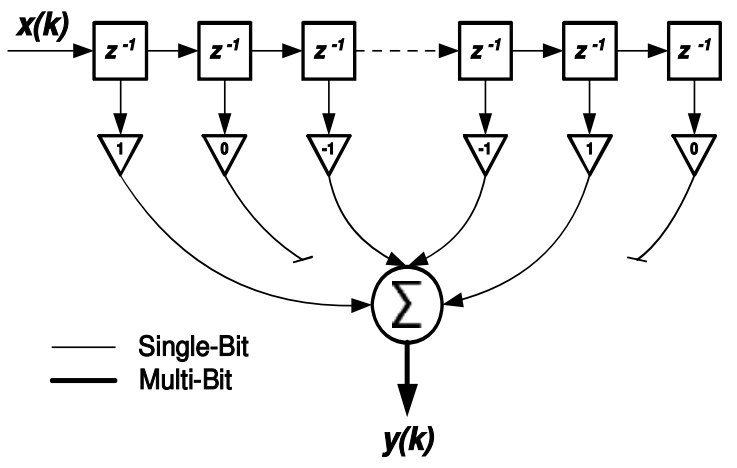

Fig. 3. Block diagram of ternary FIR filter.

\section{RECTIFICATION AND REMODULATION}

Rectification is preformed at the output of the ternary FIR filter. This rectification can be implemented with a multiplexor. The multiplexor outputs either the input signal or sets the output to zero depending on the state of the sign-bit. If the sign-bit is positive or zero it outputs the signal at the input else if the input has a negative sign-bit the multiplexor outputs zero. Hence, the rectification operation is achieved efficiently.

The multi-bit output from the rectifier is then remodulated back to single-bit. This remodulation takes place to simplify the subtraction and following multiplication as shown in Figure 2. The double loop $\Sigma \Delta \mathrm{m}$ is used to remodulate the signal because it performs well and has a simple implementation that contains only summers, delays and a quantizer. The structure of the double loop $\Sigma \Delta \mathrm{m}$ used in the BFSK demodulator is shown in figure 4. The system transfer function is given in equation 2 . 


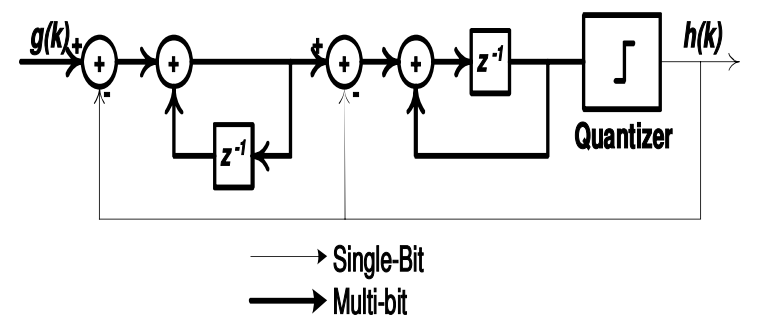

Fig. 4. Block diagram of the double loop $\Sigma \Delta \mathrm{m}$.

\subsection{Subtraction and Remodulation}

After remodulation to single-bit the two frequency branches are subtracted. This produces a ternary output that if left will overload the final $\Sigma \Delta \mathrm{m}$ 's quantizer. To stop this overloading a gain element $g$ is utilized. The gain was set to 0.5 since, the only possible outputs of the subtraction operation are $\{2,0,-2\}$. This gain will ensure that the output will never traverse above the absolute maximum quantizer threshold of 1 . The multiplication of the subtractors output can be done with a simple lookup table as one multiplicand is fixed and the other is restricted to the ternary set $\{2,0,-2\}$. The subtraction and gain functions can be achieved efficiently by using one simple two input lookup table.

\section{RESULTS AND DISCUSSION}

As proof of concept and to illustrate the capabilities of the single-bit non-coherent BFSK demodulator simulations in simulink were undertaken. The signal to noise ratio (SNR) of an additive white gaussian noise (AWGN) channel was varied to see the effect on the probability of error at the demodulator output.

Before simulations can begin the bandpass ternary filters taps are required. The method in [2] was used to generate the taps. Firstly, the remez exchange algorithm was used to generate a target frequency response. This response was then remodulated by the double loop $\Sigma \Delta \mathrm{m}$ to provide the ternary taps for the simulation. The resultant frequency response of the two ternary filters is shown in Figure 5. The separation of the bandpass center frequencies was set at $1000 \mathrm{~Hz}$. The center frequencies of the bandpass filters was set to $f_{1}=2000 \mathrm{~Hz}$ and $f_{2}=3000 \mathrm{~Hz}$ and the filter passband width is $W_{f}=650 \mathrm{~Hz}$. The stopband attenuation was set at $48 \mathrm{db}$. The stopband attenuation was set to match an input SNR capable from and 8-bit analog to digital converter, i.e. $48 \mathrm{~dB}$.

The simulation was performed at an oversampling ratio of 128 and the symbol rate was set as 1000 samples per second. One hundred thousand symbols were passed through the single-bit BFSK demodulator at a number of different SNR and the resultant output was recorded. The SNR was set via the symbol energy to noise ratio $\left(E_{s} / N_{o}\right)$. The output of the demodulator is in the single-bit format. To determine the error performance of the single-bit demodulator it is neces-

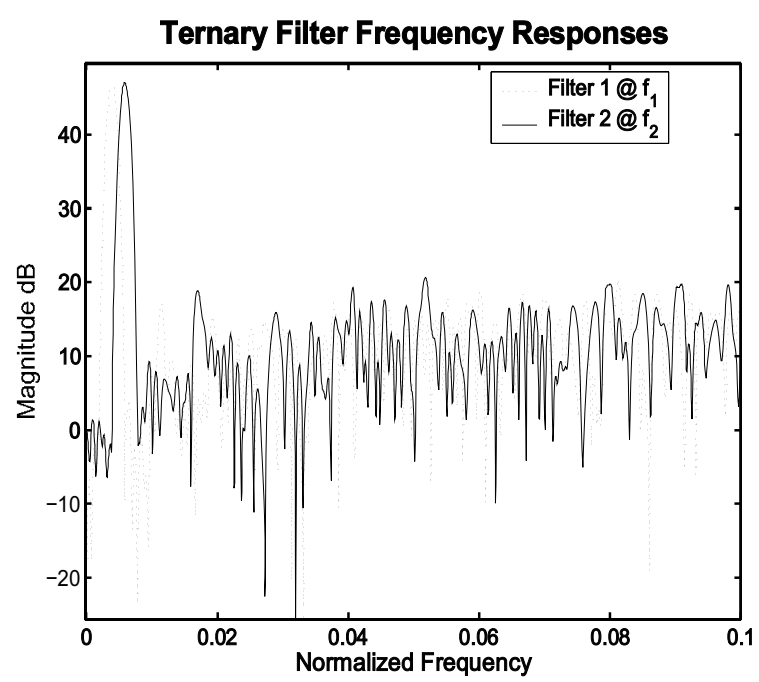

Fig. 5. Frequency response of the bandpass ternary taps. The center frequencies of the bandpass filters are $f_{1}=2000$ $\mathrm{Hz}$ and $f_{2}=3000 \mathrm{~Hz}$, for filter no. 1 and no. 2 respectively.

sary to place the demodulator output in the baseband binary format. This is achieved by lowpass filtering the single-bit BFSK output and passing the filtered signal through a threshold detector. The symbol decision threshold was set at the middle the symbol period to complete the transformation to the baseband binary format. The original baseband signal and the demodulated signal are compared to determine the bit error rate (or the probability of error $P_{e}$ ) of the demodulator. An example of the binary input signal is shown in Figure 6 in the upper graph. The single-bit demodulator outputs are shown in the lower graph in Figure 6. The filtered output has been scaled for the reader to clearly show the filtered output.

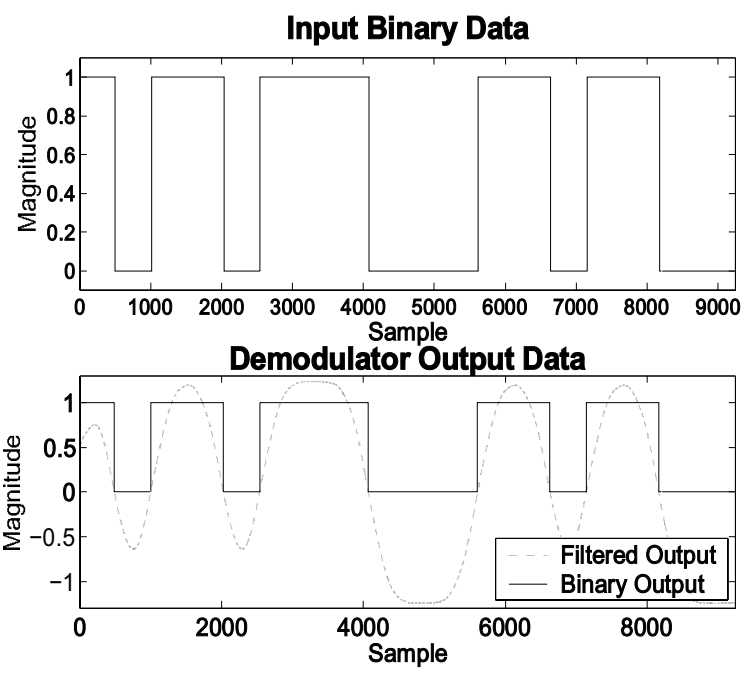

Fig. 6. Example of single-bit BFSK demodulator input and output waveforms. SNR $=20 \mathrm{~dB}$, symbol rate $=1000 \mathrm{sym}$ bols/sec.

The graph of the the total demodulator errors and 


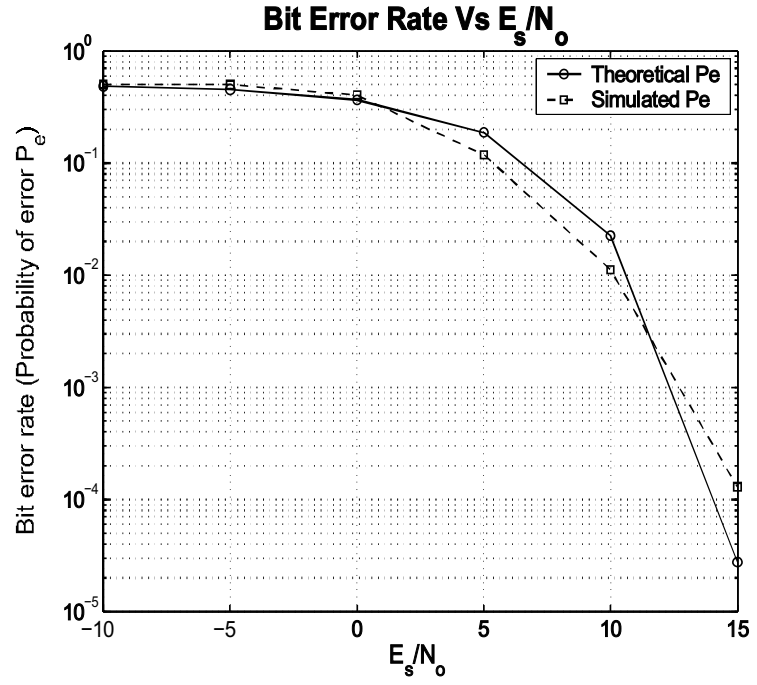

Fig. 7. Bit error rate Vs symbol to noise powers ratio.

total number of sample ratio verses symbol energy to noise power ratio is shown in Figure 7. As a comparison, the theoretical probability of error $\left(P_{e}\right)$ for a non-coherent BFSK demodulator is also plotted [10]. The equation of the $P_{e}$ theoretical line is:

$$
P_{e}=\frac{1}{2} \exp \left(-\frac{A^{2}}{4 N_{o} W_{f}}\right)
$$

where $\mathrm{A}$ is the input sinusoid amplitude, $N_{o}$ is the power spectral density of the input noise and $W_{f}$ is the bandwidth of the bandpass filters.

The simulated and theoretical graphs in Figure 7 show good correlation, particularly at the lower $E_{s} / N_{o}$. The theoretical graph shows the probability of error being lower at $E_{s} / N_{o}=15 \mathrm{~dB}$. The cause of this deviation may be due to in-band noise created from the remodulation operations within the single-bit demodulator. This effect may be reduced if the system sampling frequency is increased. Alternatively a $\Sigma \Delta \mathrm{m}$ with better noise shaping ability may also improve the bit error rate at higher $E_{s} / N_{o}$ ratios. These options requires further investigation. The authors also found that increasing the symbol sample rate above 1000 samples/sec caused an increase in the bit error rate at the demodulator output.

\section{CONCLUSIONS}

A non-coherent digital single-bit binary frequency shift keying demodulator was presented. Through the use of single-bit processing techniques this new structure was able to successfully demodulate BFSK signals with a single-bit output. The structure, although similar to the traditional non-coherent BFSK demodulator, contains no multi-bit multiplications. This greatly reduces the implementation complexity of the single-bit system.

The demodulator performance was briefly investigated through the use of an AWGN channel and determination of errors at the demodulators output. The signal energy to noise power ratio was varied from 15 to $-10 \mathrm{~dB}$ to describe the error performance with an increasingly noise degraded signal. The simulated and theoretical graphs show good correlation at lower $E_{s} / N_{o}$. Further investigation is required to determine the bit error rates at different symbol rates and with different oversampling ratios.

\section{REFERENCES}

[1] Edited By S. R. Norsworthy, R. Schreier, Gabor C. Temes, "Delta-Sigma data converters: theory, design, and simulation" IEEE Press, 1997.

[2] A. C. Thompson, P. O'Shea, Z. M. Hussain, and B. R. Steele, "Efficient single-bit ternary digital filtering using sigma-delta modulator", "Signal Processing Letters, IEEE”, vol. 11, no. 2, pp. 164-166, Feburary 2004.

[3] H. Fujisaka, R. Kurata, M. Sakamoto and M. Morisue, "Bit-stream signal processing and its application to communication systems" IEE Proceedings Circuits Devices and Systems, vol. 149, no. 3, pp. 159-166, Jun. 2002.

[4] C. Dick, F. Harris, "FPGA signal processing using sigma-delta modulation", Signal Processing Magazine, IEEE, vol. 17, no 1, pp. 20-35, 2000.

[5] David A. Johns and David M. Lewis, "Design and Analysis of Delta-Sigma Based IIR Filters," IEEE Transactions on Circuits and Systems -II: Analog and Digital Signal Processing, vol. 40, no. 4, pp. 233-240, April 1993.

[6] B. Steele and P. O'Shea, "Design of ternary digital filters" Proceedings of the Third International Conference on Information, Communications and Signal Processing (ICICS'2001), October 2001.

[7] S. M. Kershaw, S. Summerfield, M. B. Sandler, and M. Anderson, "Realisation and implementation of a sigma-delta bit stream FIR filter", IEE Proc.-Circuits, Devices, and Systems, vol. 143, no. 5, October 1996.

[8] Ping Wah Wong, "Fully sigma-delta modulation encoded FIR filters" IEEE Transactions on Signal Processing, vol. 40, no. 6, pp. 1605-1610, June 1992.

[9] Ping Wah Wong, Robert M. Gray, "Fir Filters with Sigma-Delta Modulation Encoding" IEEE Transactions on Acoustics, Speech. and Signal Processing, Vol. 38, No. 6, June, 1990, pp. 979-990

[10] Bernard Sklar, "Digital communications fundamentals and applications" Prentice Hall PTR, 2001. 